Brown-HET-1612

NSF-KITP-11-076

\title{
Symbols of One-Loop Integrals From Mixed Tate Motives
}

\author{
Marcus Spradlin and Anastasia Volovich \\ Brown University, Providence, RI 02912, USA and \\ Kavli Institute for Theoretical Physics, \\ University of California, Santa Barbara, CA 93106, USA
}

\begin{abstract}
We use a result on mixed Tate motives due to Goncharov [1] to show that the symbol of an arbitrary one-loop $2 m$-gon integral in $2 m$ dimensions may be read off directly from its Feynman parameterization. The algorithm proceeds via recursion in $m$ seeded by the well-known box integrals in four dimensions. As a simple application of this method we write down the symbol of a three-mass hexagon integral in six dimensions.
\end{abstract}




\section{MOTIVATION}

Recent breathtaking advances in our understanding of the mathematical structure of scattering amplitudes ${ }^{1}$ in maximally supersymmetric Yang-Mills theory (SYM) have so far had the most dramatic impact on tree-level amplitudes or quantities (such as leading singularities or the planar integrand [5]) which are completely determined in terms of tree-level data. The guiding principle behind these advances is the observation that amplitudes exhibit simplicity which is completely obscured by their traditional Feynman diagram expansions.

In contrast the problem of actually evaluating the integrals which appear in multi-loop amplitudes remains extremely difficult in general (see [6] for an introduction to modern methods). However data is starting to accumulate which suggests that the final results for amplitudes (or even individual integrals) can again be far simpler than one might have guessed on the basis of results obtained from more traditional integration approaches, such as Mellin-Barnes techniques.

In [7] Goncharov, Vergu and the authors introduced to the SYM literature a powerful mathematical tool first developed in [8 10], called the symbol of a transcendental function, for analyzing functions of the type which appear in all currently known SYM loop amplitudes $^{2}$. The symbol serves as a sort of motivic roadmap, encoding in a simple way all of the information about a function's discontinuities without the need to introduce any explicit representation of the function in terms of (generalized) polylogarithm functions. Indeed one might reasonably say that the symbol encapsulates all of the physically relevant information that one may want to know about any given integral or amplitude. We view the symbol as stepping stone halfway between an integrand and its integral, and it is our hope that it may help to guide us through the dark jungle of multi-loop amplitudes.

The simple formula given in [7] for the two-loop six-point maximally helicity violating (MHV) remainder function was made possible by applying motivic technology to the 17-page analytic expression which had been heroically evaluated by Del Duca, Duhr and Smirnov [16, 17]. It is clear that we are now desperately in need of some technology which would allow one to directly compute the symbol of any given integral without the need for such heroic effort. Since the planar integrand of SYM is a rational form, and since the symbol of an

\footnotetext{
${ }^{1}$ See for example [2-4] for reviews and recent progress.

${ }^{2}$ See [4, 11 15] for recent applications of symbol technology.
} 
integral involves only rational functions, it is reasonable to suppose that there should exist an algebraic operation which would allow one to simply write down the symbol of any given integrand. This would provide a concrete realization of part of the ambitious program suggested in [8] (see in particular section 7) which aims for a "correspondence principle" unifying scattering amplitudes, their motivic avatars, and the combinatorics of Feynman diagrams reflected in the coproduct formula. There too the symbol is not the ultimate goal but serves as just the simplest incarnation of the amplitudes and their motivic structure.

Work towards unlocking the structure of general multi-loop integrals is underway, but given the recent flurry of activity [12-15] on one-loop hexagon integrals in six dimensions we feel compelled to focus in this brief note on the special case of the one-loop $2 m$-gon integral in $2 m$ dimensions. The motivic structure of this integral is sufficiently simple that its symbol can be read off directly from its Feynman parameterization by utilizing a 1996 theorem on mixed Tate motives due to A. Goncharov. These higher dimensional integrals have several applications in physics, for example they appear in dimensionally-regulated one-loop MHV

amplitudes [18-20] and in certain cases they can be related to higher-loop four-dimensional integrals [21, 22].

In section II we set up some notation. The main result is presented in section III, followed by several sample applications in section IV, including a particular three-mass hexagon integral in six dimensions. Note that in this note we work entirely at the level of symbols and do not address the problem of choosing an explicit representative for integrals in terms of (generalized) polylogarithm functions. It is our understanding that efforts to automate this final step are under way [23].

\section{MOTIVIC AVATARS OF ONE-LOOP SCALAR INTEGRALS}

In this note we consider the one-loop $2 m$-gon integral in $2 m$ dimensions, specifically

$$
F_{m}\left(y_{i}\right)=\Delta \int \frac{d^{2 m} y}{(i \pi)^{m}} \prod_{i=1}^{2 m} \frac{1}{\left(y-y_{i}\right)^{2}}
$$

considered as a function of $2 m$ points $y_{1}, \ldots, y_{2 m}$ (with an implied cyclic ordering) in $2 m$ dimensional Minkowski space. We have chosen to include in this definition the prefactor

$$
\Delta=\sqrt{(-1)^{m} \operatorname{det} y_{i j}^{2}}, \quad y_{i j}=y_{i}-y_{j}
$$


which serves three intimately related purposes: it ensures that $F_{m}\left(y_{i}\right)$ is invariant under dual conformal transformations [24, 25], it normalizes the leading singularities of the integrand to \pm 1 , and it ensures that $F_{m}$ evaluates to a function of well-defined transcendentality $m$. (We caution the reader that our $\Delta$ is close, but not exactly equal, to the quantity called $\Delta$ in $[12,13]$.

Results for the $m=2$ integrals have of course been known since antiquity (see for example [27] for a comprehensive modern treatment), while only very recently the first two cases of $m=3$ have been studied: the massless hexagon in [12,13] and the one-mass hexagon in [14]. We remind the reader that the integral (11) is said to be massless if cyclically adjacent pairs are all null-separated, i.e. $y_{i, i+1}^{2}=0$ for all $i$. More generally we say that the integral has $k$ masses if $k$ pairs of cyclically adjacent points are null-separated. For $m>2$ the integral $F_{m}\left(y_{i}\right)$ always converges, while for $m=2$ (the case of box integrals in four dimensions) it only converges in the fully massive case. We will return to the question of divergences in detail below.

The $d^{2 m} y$ integral is easily performed after introducing Feynman parameters to combine the $2 m$ propagators, which leads to

$$
F_{m}\left(y_{i}\right)=\Gamma(m) \Delta \int_{0}^{\infty} d^{2 m} \alpha \frac{\delta\left(\alpha_{1}-1\right)}{\left(\sum_{i<j} y_{i j}^{2} \alpha_{i} \alpha_{j}\right)^{m}}
$$

One may choose any nontrivial linear combination of the $\alpha$ 's inside the delta-function [6] but our simple choice of $\alpha_{1}$ makes it immediately manifest that (3) is best thought of as a projective integral ${ }^{3}$. Specifically (3) is an integral over $\mathbb{C P}^{2 m-1}$ with homogeneous coordinates $\left[\alpha_{1}: \cdots: \alpha_{2 m}\right]$ which has been written in the patch $\alpha_{1}=1$.

Therefore, the one-loop $2 m$-gon integral in $2 m$ dimensions is the $\mathbb{C P}^{2 m-1}$ period integral ${ }^{4}$

$$
F_{m}(Q)=\Gamma(m) \int D^{2 m-1} W \frac{\Delta}{\left(\frac{1}{2} W \cdot Q \cdot W\right)^{m}}, \quad \Delta=\sqrt{(-1)^{m} \operatorname{det} Q}
$$

where $D^{2 m-1} W$ is the standard holomorphic form

$$
D^{2 m-1} W=\epsilon^{i_{1} \cdots i_{2 m}} w_{i_{1}} d w_{i_{2}} \wedge \cdots \wedge d w_{i_{2 m}}
$$

and where we now use the notation $F_{m}(Q)$ to emphasize that $F_{m}$ should be thought of as a function of a quadratic form $Q$, whose matrix entries are $Q_{i j}=\left(y_{i}-y_{j}\right)^{2}$ in our application.

\footnotetext{
3 We thank N. Arkani-Hamed for emphasizing this point.

4 See [28] for a general discussion of the relation between Feynman integrals and periods.
} 
Integrals of the type (44) have been studied in the context of mixed Tate motives, and belong to a class of objects called Tate iterated integrals in [26] (see especially section 3). In particular $F_{m}(Q)$ is a special case of the more general period integrals $v(Q, M)$ studied in [1]. These are integrals of the type (4) involving any non-degenerate quadric $Q$ and integrated over a cycle representing a generator of $H_{2 m-1}\left(\mathbb{C P}^{2 m-1}, M\right)$ for an arbitrary simplex $M$ in general position relative to $Q$. The cohomology group $H^{2 m-1}\left(\mathbb{C P}^{2 m-1} \backslash Q, M ; \mathbb{Q}\right)$ has canonical mixed Hodge-Tate structure which corresponds to a certain mixed Tate motive [1].

In our particular application we shall only be interested in quadratic forms $Q$ with vanishing diagonal entries (since obviously $y_{i i}^{2}=0$ for all $i$ ). Moreover, the cycle of integration of interest in (4), inherited from the identification with (3), involves the particularly simple simplex $M$ whose $2 m$ faces are just the coordinate hyperplanes $M_{1}, \ldots, M_{2 m}$. These two simplifications of our particular $F_{m}(Q)$ 's compared to the more general $v(Q, M)$ 's studied in [1] are not of essential mathematical importance but greatly streamline the bookkeeping involved in applying the results of that paper.

\section{AN AUTO-MOTIVE RECURSION FOR ONE-LOOP SYMBOLS}

We will now write a simple recursive formula for the symbol $S_{m}(Q)$ of $F_{m}(Q)$. The recursion expresses $S_{m}(Q)$ as a sum of $m(2 m-1)$ terms involving $S_{m-1}\left(Q_{\overline{i j}}\right)$, where the notation $Q_{\overline{i j}}$ denotes the $(2 m-2) \times(2 m-2)$ matrix obtained by deleting the rows and columns $i$ and $j$ from the $2 m \times 2 m$ matrix $Q$ (i.e., the quadric $Q_{\overline{i j}}$ is just the intersection $\left.Q \cap M_{i} \cap M_{j}\right)$. In terms of the Feynman graph corresponding to the one-loop integral, going from $Q$ to $Q_{\overline{i j}}$ amounts to turning the $2 m$-gon into a $2 m-2$-gon by deleting the two propagators $\left(y-y_{i}\right)^{2}$ and $\left(y-y_{j}\right)^{2}$. There are $m(2 m-1)$ different ways of doing this, and each way leads to one term in the recursive sum.

To obtain the recursion we apply Theorem (4.10) of [1] (preprint version), which for us becomes

$$
d F_{m}(Q)=\frac{1}{2} \sum_{i<j} F_{m-1}\left(Q_{\overline{i j}}\right) d \log R_{i j}
$$

where

$$
R_{i j}=\frac{Q_{i j}^{-1}+\sqrt{\left(Q_{i j}^{-1}\right)^{2}-Q_{i i}^{-1} Q_{j j}^{-1}}}{Q_{i j}^{-1}-\sqrt{\left(Q_{i j}^{-1}\right)^{2}-Q_{i i}^{-1} Q_{j j}^{-1}}}
$$


Note that no summation of indices is implied on the right-hand side, which is expressed in terms of the $i i, i j$ and $j j$ entries of the inverse matrix $Q^{-1}$.

Let us comment briefly on the geometric content of (7). For each choice of $i$ and $j$, there are precisely two codimension-1 hyperplanes $H_{1}, H_{2}$ in $\mathbb{C P}^{2 m-1}$ which contain the intersection $M_{i} \cap M_{j}$ and which are tangent to the quadric $Q=\left\{W \in \mathbb{C P}^{2 m-1}: \frac{1}{2} W \cdot Q \cdot W=0\right\}$. So for each $i$ and $j$ we have four natural codimension-1 hyperplanes $\left\{M_{i}, M_{j}, H_{1}, H_{2}\right\}$ all of which contain $M_{i} \cap M_{j}$ as a common codimension-2 subspace, and the quantity $R_{i j}$ in (7) is the cross-ratio naturally associated to this configuration.

It follows immediately from (6) that the symbol $S_{m}(Q)$ satisfies the recursion

$$
S_{m}(Q)=\frac{1}{2} \sum_{i<j} S_{m-1}\left(Q_{\overline{i j}}\right) \otimes R_{i j}
$$

In what follows we shall only make use of (하), though of course (66) is a slightly stronger statement which realizes a precise set of differential relations between the infinite class of oneloop $2 m$-gon integrals. Various types of differential relations amongst multi-loop integrals have long been studied; see [13, 21] for some recent examples which also relate integrals in different dimensions.

\section{SAMPLE APPLICATIONS OF THE MB (MOTIVIC BEAUTY) TECHNIQUE}

We begin with a couple of almost trivial examples demonstrating the application of the recursion (8). In general mathematical applications one may seed the recursion with the result of the $\mathbb{C P}^{1}$ integral

$$
F_{1}(Q)=\int D^{1} W \frac{\Delta}{\left(\frac{1}{2} W \cdot Q \cdot W\right)}=\log \frac{Q_{12}+\Delta}{Q_{12}-\Delta}, \quad \Delta=\sqrt{-\operatorname{det} Q} .
$$

However in physics applications, where we are always interested in quadrics $Q$ with zero entries down the diagonal, this 'bubble' integral is too singular to be of use by itself.

Instead it is more effective to seed the recursion with box integrals $(m=2)$. As mentioned above these are divergent except in the fully massive case. Nevertheless, as we discuss in detail below, it is relatively straightforward to apply this recursion to arbitrary integrals by introducing if necessary a regularization parameter $\epsilon$ which is ultimately taken to zero. The finiteness of the integral $F_{m}(Q)$ for $m>2$ is reflected in the fact that the regularization 
parameter always drops completely out of the symbol $S_{m}(Q)$ after summing together all contributions to (8) .

Before proceeding let us make an important comment regarding notation. In the previous section we defined the integral $F_{m}\left(y_{i}\right)$ for an arbitrary collection of $2 m$ points $y_{i}$ in Minkowski space, without regard to whether or not any cyclically adjacent pairs were null separated. In the remainder of this paper we shall adopt notation more conventional in the physics literature, whereby we consider a collection of $n \geq 2 m$ points which are all mutually null, i.e. $\left(x_{i}-x_{i+1}\right)^{2}=0$ for all $i$, but only a subset of $2 m$ of these $x_{i}$ 's will be chosen as the $2 m$ arguments to the function $F_{m}\left(y_{i}\right)$.

\section{A. The Four-Mass Box Integral}

Let us start with the four-mass box integral, which corresponds to the quadric

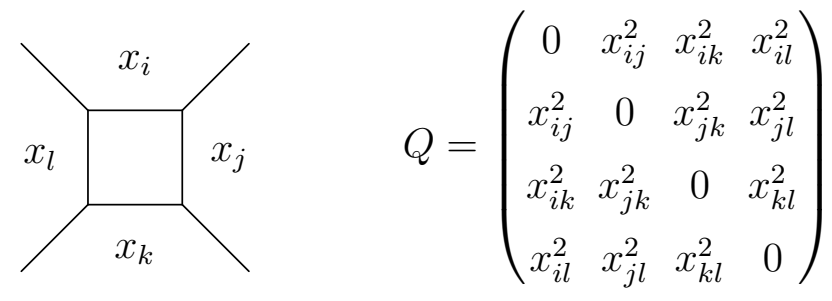

in $\mathbb{C P}^{3}$. The recursion (8) expresses the symbol of this integral as a sum over six $\mathbb{C P}^{1}$ integrals of the type 9):

$$
\begin{aligned}
S_{2}(Q)=\frac{1}{2} & \left(S_{1}\left(Q_{\overline{12}}\right) \otimes R_{12}+S_{1}\left(Q_{\overline{13}}\right) \otimes R_{13}+S_{1}\left(Q_{\overline{14}}\right) \otimes R_{14}\right. \\
& \left.+S_{1}\left(Q_{\overline{23}}\right) \otimes R_{23}+S_{1}\left(Q_{\overline{24}}\right) \otimes R_{24}+S_{1}\left(Q_{\overline{34}}\right) \otimes R_{34}\right) .
\end{aligned}
$$

Using (9) we find for example

$$
S_{1}\left(Q_{\overline{12}}\right)=\frac{x_{k l}^{2}+\sqrt{x_{k l}^{4}}}{x_{k l}^{2}-\sqrt{x_{k l}^{4}}}, \quad R_{12}=\frac{x_{i l}^{2} x_{j k}^{2}+x_{i k}^{2} x_{j l}^{2}-x_{i j}^{2} x_{k l}^{2}-\Delta}{x_{i l}^{2} x_{j k}^{2}+x_{i k}^{2} x_{j l}^{2}-x_{i j}^{2} x_{k l}^{2}+\Delta}
$$

where $\Delta=\sqrt{+\operatorname{det} Q}$ in this case.

Evidently the symbol $S_{1}\left(Q_{\overline{12}}\right)$ is singular, but this can be regulated by replacing all of the zero entries on the diagonal of $Q$ with $\epsilon$. Then, at the level of the symbol, we can replace

$$
S_{1}\left(Q_{\overline{12}}(\epsilon)\right)=\frac{x_{k l}^{2}+\sqrt{x_{k l}^{4}-\epsilon^{2}}}{x_{k l}^{2}-\sqrt{x_{k l}^{4}-\epsilon^{2}}}=\frac{x_{k l}^{4}}{\epsilon^{2}}+\mathcal{O}(1) \Longrightarrow \frac{x_{k l}^{4}}{\epsilon^{2}}
$$


Here we have freely dropped an overall factor of 4 since such numerical constants drop out of the symbol. Note that we could have introduced the $\epsilon$ into $R_{12}$ as well but there is no need as this quantity has a finite limit, shown above, as $\epsilon \rightarrow 0$.

Adding up the six contributions (11) we find first of all that $\epsilon$ completely drops out of the symbol (an important consistency check), and that the result can be assembled into the form

$$
\begin{aligned}
S_{2}(Q) & =x_{i l}^{2} x_{j k}^{2} \otimes \frac{-x_{i l}^{2} x_{j k}^{2}+x_{i k}^{2} x_{j l}^{2}+x_{i j}^{2} x_{k l}^{2}-\Delta}{-x_{i l}^{2} x_{j k}^{2}+x_{i k}^{2} x_{j l}^{2}+x_{i j}^{2} x_{k l}^{2}+\Delta} \\
& +x_{i k}^{2} x_{j l}^{2} \otimes \frac{+x_{i l}^{2} x_{j k}^{2}-x_{i k}^{2} x_{j l}^{2}+x_{i j}^{2} x_{k l}^{2}-\Delta}{+x_{i l}^{2} x_{j k}^{2}-x_{i k}^{2} x_{j l}^{2}+x_{i j}^{2} x_{k l}^{2}+\Delta} \\
& +x_{i j}^{2} x_{k l}^{2} \otimes \frac{+x_{i l}^{2} x_{j k}^{2}+x_{i k}^{2} x_{j l}^{2}-x_{i j}^{2} x_{k l}^{2}-\Delta}{+x_{i l}^{2} x_{j k}^{2}+x_{i k}^{2} x_{j l}^{2}-x_{i j}^{2} x_{k l}^{2}+\Delta}
\end{aligned}
$$

which of course agrees precisely with the symbol of the well-known four-mass box function

$$
F_{2}=\operatorname{Li}_{2}\left(x_{+} / x_{-}\right)-\operatorname{Li}_{2}\left(\frac{1-x_{+}}{1-x_{-}}\right)+\operatorname{Li}_{2}\left(\frac{1-1 / x_{+}}{1-1 / x_{-}}\right)-\left(x_{+} \leftrightarrow x_{-}\right)
$$

in terms of

$$
x_{ \pm}=\frac{1}{2} \frac{x_{i l}^{2} x_{j k}^{2}+x_{i k}^{2} x_{j l}^{2}-x_{i j}^{2} x_{k l}^{2} \pm \Delta}{x_{i l}^{2} x_{j k}^{2}} .
$$

\section{B. Other Box Integrals in Four Dimensions}

From a mathematical point of view it is perhaps simplest to restrict one's attention to the generic case of the completely massive $2 m$-gon integral, whose symbol may now be written down directly using the recursion (8) seeded by (14) at $m=2$.

However in physics we are often interested in integrals with fewer masses. The application of the recursion (8) in these cases will call into play various divergent box integrals which can be regulated in a manner similar to (13). For example consider the three-mass box integral with corresponding quadric

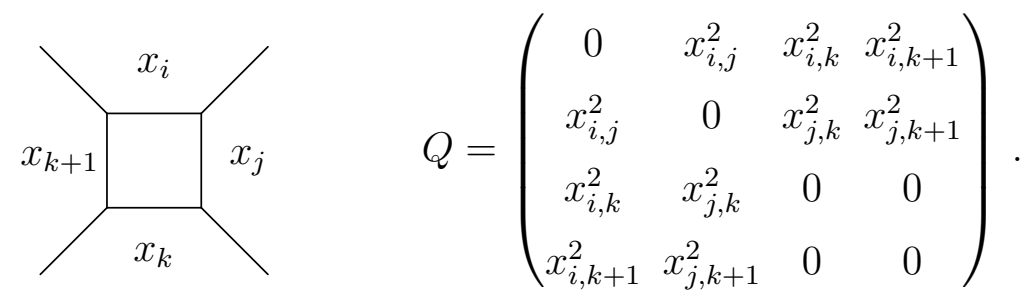


Its symbol may be obtained by setting $x_{k l}^{2}=\epsilon$ in (14) and keeping only the leading-order terms for small $\epsilon$, which leads to

$$
\begin{aligned}
S_{2}(Q)=\frac{1}{2}\left(\frac{\epsilon^{2} x_{i, j}^{4}}{x_{i, k}^{2} x_{i, k+1}^{2} x_{j, k}^{2} x_{j, k+1}^{2}} \otimes \frac{x_{i, k}^{2} x_{j, k+1}^{2}}{x_{i, k+1}^{2} x_{j, k}^{2}}\right. & \left.+\frac{x_{i, k}^{2} x_{j, k+1}^{2}}{x_{i, k+1}^{2} x_{j, k}^{2}} \otimes \frac{\epsilon^{2} x_{i, j}^{4}}{x_{i, k}^{2} x_{i, k+1}^{2} x_{j, k}^{2} x_{j, k+1}^{2}}\right) \\
& +\frac{x_{i, k+1}^{2} x_{j, k}^{2}}{x_{i, k}^{2} x_{j, k+1}^{2}} \otimes \frac{\left(x_{i, k}^{2} x_{j, k+1}^{2}-x_{i, k+1}^{2} x_{j, k}^{2}\right)^{2}}{x_{i, k}^{2} x_{i, k+1}^{2} x_{j, k}^{2} x_{j, k+1}^{2}} .
\end{aligned}
$$

Results for all other box integrals may be obtained as further specializations of this formula. For example the two-mass easy or two-mass hard box functions can be obtained by setting $x_{i j}^{2}=\epsilon$ or $x_{j k}^{2}=\epsilon$ respectively. Note that if we use (14) as our starting point then it is not necessary to introduce an $\epsilon$ for each of the diagonal entries of $Q$; in writing (14) all of those divergences have already been properly eliminated. We only need to introduce an $\epsilon$ for any zero entry immediately adjacent to the diagonal (i.e. for any massless vertex of the corresponding diagram).

Just like we saw in (14), all dependence on the regularization parameter $\epsilon$ will necessarily drop out when individually divergent contributions are properly assembled to produce the symbol of any of the finite functions $F_{m}$ for $m>2$. In practice this cancellation can serve as a useful check against calculational errors.

\section{A Three-Mass Hexagon in Six Dimensions}

As our first non-trivial ${ }^{5}$ demonstration of the main result let us write the symbol for the three-mass hexagon integral in six dimensions corresponding to the quadric

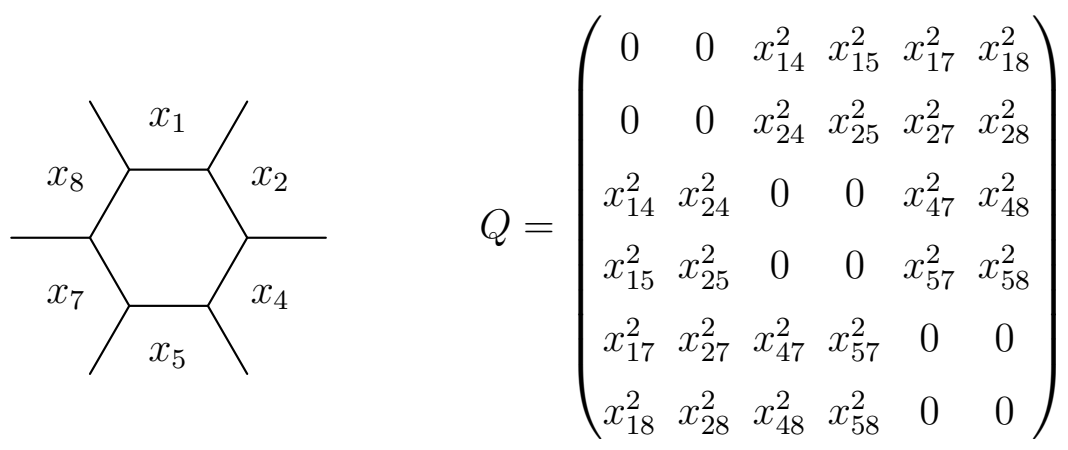

\footnotetext{
${ }^{5}$ Actually in this particular case it seems possible to generate the symbol directly from the Feynman parameterization (3) with the help of Mathematica's Integrate command; in our experience it generally gets stuck starting at degree 4 where the classical polylogarithms do not provide a sufficiently large basis of functions.
} 
in $\mathbb{C P}^{5}$. Here we have chosen for convenience an integral with $n=9$ particles, which manifestly exhibits a $\mathbb{Z}_{3}$ symmetry.

We shall for convenience restrict the external kinematics to four dimensions (i.e. all of the $x_{i}$ lie within a common four-dimensional subspace of six-dimensional Minkowski space). This allows us to express everything in terms of momentum twistors [29]; in particular $x_{i j}^{2} \propto\langle i i+1 j j+1\rangle$ where the constant of proportionality is irrelevant as it cancels out in all conformally invariant quantities.

We make this choice for purposes of notational simplicity only but we emphasize that the result (8) is of course valid for completely general kinematics ${ }^{6}$. A special feature of the three-mass hexagon (19) is that no square roots of momentum twistor invariants appear in its symbol. This would not be true if any two adjacent vertices of the hexagon were massive.

The recursion (8) expresses the symbol $S_{3}(Q)$ as a sum of 15 terms, but we can use the $\mathbb{Z}_{3}$ symmetry of this configuration to cast the result into the form ${ }^{7}$

$$
\begin{aligned}
\left(1+g+g^{2}\right) & {\left[S_{2}\left(Q_{\overline{12}}\right) \otimes \frac{\langle 2358\rangle\langle 12 \overline{5} \cap \overline{8}\rangle}{\langle 1258\rangle\langle 23 \overline{5} \cap \overline{8}\rangle}\right.} \\
& +S_{2}\left(Q_{\overline{13}}\right) \otimes \frac{\langle 1238\rangle\langle 2568\rangle\langle 5789\rangle\langle 23 \overline{5} \cap \overline{8}\rangle}{\langle 2358\rangle\langle 2789\rangle\langle 4568\rangle\langle 56 \overline{2} \cap \overline{8}\rangle} \\
& +S_{2}\left(Q_{\overline{14}}\right) \otimes \frac{\langle 2358\rangle\langle 2789\rangle\langle 4568\rangle\langle 45 \overline{2} \cap \overline{8}\rangle}{\langle 1238\rangle\langle 2458\rangle\langle 5789\rangle\langle 23 \overline{5} \cap \overline{8}\rangle} \\
& +S_{2}\left(Q_{\overline{16}}\right) \otimes \frac{\langle 2358\rangle\langle 2456\rangle\langle 5789\rangle\langle 78 \overline{2} \cap \overline{5}\rangle}{\langle 1235\rangle\langle 2578\rangle\langle 4568\rangle\langle 23 \overline{5} \cap \overline{8}\rangle} \\
& \left.+S_{2}\left(Q_{\overline{24}}\right) \otimes \frac{\langle 1238\rangle\langle 2458\rangle\langle 5789\rangle\langle 12 \overline{5} \cap \overline{8}\rangle}{\langle 1258\rangle\langle 2789\rangle\langle 4568\rangle\langle 45 \overline{2} \cap \overline{8}\rangle}\right]
\end{aligned}
$$

where $g: i \rightarrow i+3$ is the shift-by-three operator and we use the notation

$$
\langle i j \bar{k} \cap \bar{l}\rangle=\langle i k-1 k k+1\rangle\langle j l-1 l l+1\rangle-\langle j k-1 k k+1\rangle\langle i l-1 l l+1\rangle .
$$

Appearing in the first two entries of the symbol are a two-mass easy box $Q_{\overline{12}}$ and some three-mass boxes $Q_{\overline{13}}, Q_{\overline{14}}, Q_{\overline{16}}$ and $Q_{\overline{24}}$, corresponding to the degenerations of the hexagon:

\footnotetext{
${ }^{6}$ Indeed we note that with four-dimensional kinematics there does not exist any quadric integral of the form (4) for the octagon or higher since for $m>3$ the determinant $\operatorname{det} y_{i j}^{2}$ is identically zero for any collection of $2 m$ points $y_{i}$ which lie in a common four-dimensional subspace.

${ }^{7}$ A file containing the complete symbol with all terms written out explicitly is included with the arXiv submission.
} 

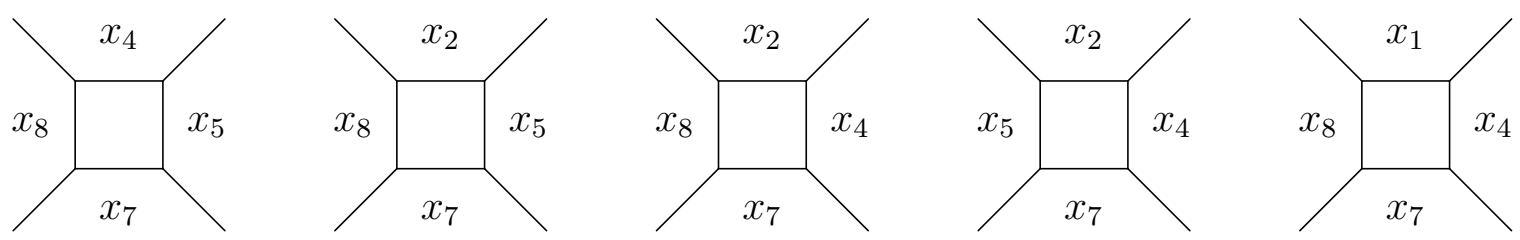

Their $\epsilon$-dependent symbols may be read off from (18), and it is a nontrivial check of the correctness of (20) that all of the $\epsilon$ dependence completely drops out at the level of the symbol. It is also straightforward to verify that in the limit $x_{24}^{2} \rightarrow 0, x_{57}^{2} \rightarrow 0$ and $x_{18}^{2} \rightarrow 0$ the result (20) reproduces the symbol of the massless hexagon computed in [12, 13].

In conclusion let us emphasize that in contrast to the old MB method, the new MB (motivic beauty) technique does not require the evaluation of any integrals. The only even slightly nontrivial step in obtaining (20) was inverting the matrix $Q$ shown in (19) and simplifying the $R_{i j}$ 's from (77) to the final form presented. Generalizations of this method to projective integrals of the type which appear in higher loop integrals remain under active investigation.

In this note we have worked entirely at the level of symbols, without addressing the important problem of finding explicit representations for integrals in terms of (generalized) polylogarithm functions. This remains an interesting open problem, especially since our experience with [7] has shown that analytic expressions for amplitudes can turn out to be much simpler than one might have guessed from their symbols.

\section{Acknowledgments}

We are grateful to S. Caron-Huot, J. Henn, D. Skinner, C. Vergu and especially N. ArkaniHamed and A. Goncharov for useful discussions, correspondence, and encouragement. This work was supported in part by the Department of Energy under contract DE-FG0291ER40688 Task J OJI (MS) and Task A (AV), and the National Science Foundation under Grant Nos. PHY05-51164, PECASE PHY-0643150 (AV) and ADVANCE 0548311 (AV).

[1] A. Goncharov, "Volumes of hyperbolic manifolds and mixed Tate motives," J. AMS 12, 569 (1999), arXiv:alg-geom/9601021. 
[2] Journal of Physics A special issue on "Scattering Amplitudes in Gauge Theories," to appear, eds. R Roiban, M. Spradlin and A. Volovich, with contributions by H. Elvang, D. Z. Freedman and M. Kiermaier, arXiv:1012.3401; R. Britto, arXiv:1012.4493; J. M. Henn, arXiv:1103.1016; Z. Bern and Y. t. Huang, arXiv:1103.1869; R. M. Schabinger, arXiv:1103.2769; A. Brandhuber, B. Spence and G. Travaglini, arXiv:1103.3477; T. Bargheer, N. Beisert and F. Loebbert, arXiv:1104.0700; J. Bartels, L. N. Lipatov and A. Prygarin, arXiv:1104.0816; T. Adamo, M. Bullimore, L. Mason and D. Skinner, arXiv:1104.2890; L. J. Dixon, arXiv:1105.0771; H. Ita and J. Drummond.

[3] N. Arkani-Hamed, J. L. Bourjaily, F. Cachazo and J. Trnka, "Local Integrals for Planar Scattering Amplitudes," arXiv:1012.6032 [hep-th].

[4] D. Gaiotto, J. Maldacena, A. Sever and P. Vieira, "Pulling the straps of polygons," arXiv:1102.0062 [hep-th].

[5] N. Arkani-Hamed, J. L. Bourjaily, F. Cachazo, S. Caron-Huot and J. Trnka, "The All-Loop Integrand For Scattering Amplitudes in Planar $N=4$ SYM," JHEP 1101, 041 (2011) arXiv:1008.2958 [hep-th]].

[6] V. Smirnov, Feynman Integral Calculus, Springer Verlag (2006).

[7] A. B. Goncharov, M. Spradlin, C. Vergu and A. Volovich, "Classical Polylogarithms for Amplitudes and Wilson Loops," Phys. Rev. Lett. 105, 151605 (2010) [arXiv:1006.5703 [hep-th]].

[8] A. B. Goncharov, "Galois symmetries of fundamental groupoids and noncommutative geometry," Duke Math J. 128, 209 (2005), arXiv:math/0208144.

[9] A. B. Goncharov "Polylogarithms and Motivic Galois groups," Proc. Symp. Pure Math. 55, 43 (1994).

[10] A. B. Goncharov, "Multiple polylogarithms, cyclotomy and modular complexes," Math. Res. Lett. 5, 497 (1998).

[11] L. F. Alday, "Some analytic results for two-loop scattering amplitudes," JHEP 1107, 080 (2011) arXiv:1009.1110 [hep-th]].

[12] V. Del Duca, C. Duhr, V. A. Smirnov, C. Duhr and V. A. Smirnov, "The massless hexagon integral in $D=6$ dimensions," Phys. Lett. B 703, 363 (2011) [arXiv:1104.2781 [hep-th]].

[13] L. J. Dixon, J. M. Drummond and J. M. Henn, "The one-loop six-dimensional hexagon integral and its relation to MHV amplitudes in $N=4$ SYM," JHEP 1106, 100 (2011) arXiv:1104.2787 [hep-th]]. 
[14] V. Del Duca, C. Duhr and V. A. Smirnov, "The One-Loop One-Mass Hexagon Integral in $D=6$ Dimensions," JHEP 1107, 064 (2011) [arXiv:1105.1333 [hep-th]].

[15] V. Del Duca, L. J. Dixon, J. M. Drummond, C. Duhr, J. M. Henn and V. A. Smirnov, "The one-loop six-dimensional hexagon integral with three massive corners," Phys. Rev. D 84, 045017 (2011) arXiv:1105.2011 [hep-th]].

[16] V. Del Duca, C. Duhr and V. A. Smirnov, "An Analytic Result for the Two-Loop Hexagon Wilson Loop in $N=4$ SYM," JHEP 1003, 099 (2010) [arXiv:0911.5332 [hep-ph]].

[17] V. Del Duca, C. Duhr and V. A. Smirnov, "The Two-Loop Hexagon Wilson Loop in $N=4$ SYM," JHEP 1005, 084 (2010) arXiv:1003.1702 [hep-th]].

[18] Z. Bern, L. J. Dixon, D. C. Dunbar and D. A. Kosower, "One loop selfdual and $N=4$ superYang-Mills," Phys. Lett. B 394, 105 (1997) [arXiv:hep-th/9611127.

[19] V. Del Duca, C. Duhr, E. W. Nigel Glover and V. A. Smirnov, "The One-loop pentagon to higher orders in epsilon," JHEP 1001, 042 (2010) arXiv:0905.0097 [hep-th]].

[20] B. A. Kniehl and O. V. Tarasov, "Analytic result for the one-loop scalar pentagon integral with massless propagators," Nucl. Phys. B 833, 298 (2010) [arXiv:1001.3848 [hep-th]].

[21] J. M. Drummond, J. M. Henn and J. Trnka, "New differential equations for on-shell loop integrals," JHEP 1104, 083 (2011) [arXiv:1010.3679 [hep-th]].

[22] C. Anastasiou and A. Banfi, "Loop lessons from Wilson loops in $N=4$ supersymmetric Yang-Mills theory," JHEP 1102, 064 (2011) arXiv:1101.4118 [hep-th]].

[23] C. Duhr, H. Gangl and J. R. Rhodes, "From polygons and symbols to polylogarithmic functions," arXiv:1110.0458 [math-ph].

[24] D. J. Broadhurst, "Summation of an infinite series of ladder diagrams," Phys. Lett. B 307, 132 (1993).

[25] J. M. Drummond, J. Henn, V. A. Smirnov and E. Sokatchev, "Magic identities for conformal four-point integrals," JHEP 0701, 064 (2007) arXiv:hep-th/0607160].

[26] A. B. Goncharov, "A simple construction of Grassmannian polylogarithms," arXiv:0908.2238 [math.AG]

[27] Z. Bern, L. J. Dixon and D. A. Kosower, "Dimensionally regulated pentagon integrals," Nucl. Phys. B 412, 751 (1994) arXiv:hep-ph/9306240.

[28] C. Bogner and S. Weinzierl, "Periods and Feynman integrals," J. Math. Phys. 50, 042302 (2009) arXiv:0711.4863 [hep-th]]. 
[29] A. Hodges, "Eliminating spurious poles from gauge-theoretic amplitudes," arXiv:0905.1473 [hep-th]. 